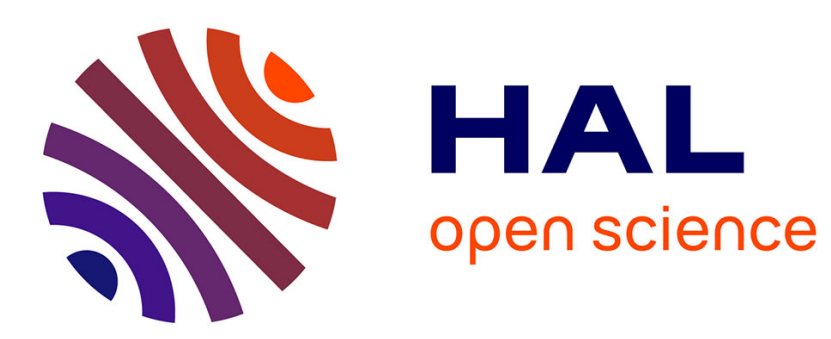

\title{
On the best observation of wave and Schrödinger equations in quantum ergodic billiards
}

\author{
Yannick Privat, Emmanuel Trélat, Enrique Zuazua
}

\section{To cite this version:}

Yannick Privat, Emmanuel Trélat, Enrique Zuazua. On the best observation of wave and Schrödinger equations in quantum ergodic billiards. Journées EDP, 2012, Biarritz, France. 13 p., 10.5802/jedp.93 . hal-00718677

\section{HAL Id: hal-00718677 \\ https://hal.science/hal-00718677}

Submitted on 17 Jul 2012

HAL is a multi-disciplinary open access archive for the deposit and dissemination of scientific research documents, whether they are published or not. The documents may come from teaching and research institutions in France or abroad, or from public or private research centers.
L'archive ouverte pluridisciplinaire HAL, est destinée au dépôt et à la diffusion de documents scientifiques de niveau recherche, publiés ou non, émanant des établissements d'enseignement et de recherche français ou étrangers, des laboratoires publics ou privés. 


\title{
On the best observation of wave and Schrödinger equations in quantum ergodic billiards
}

\author{
Yannick Privat* Emmanuel Trélat ${ }^{\dagger} \quad$ Enrique Zuazua ${ }^{\ddagger}$
}

\begin{abstract}
This paper is a proceedings version of the ongoing work [20], and has been the object of the talk of the second author at Journées EDP in 2012 .

In this work we investigate optimal observability properties for wave and Schrödinger equations considered in a bounded open set $\Omega \subset \mathbb{R}^{n}$, with Dirichlet boundary conditions. The observation is done on a subset $\omega$ of Lebesgue measure $|\omega|=L|\Omega|$, where $L \in(0,1)$ is fixed. We denote by $\mathcal{U}_{L}$ the class of all possible such subsets. Let $T>0$. We consider first the benchmark problem of maximizing the observability energy $\int_{0}^{T} \int_{\omega} \mid y(t, x)^{2} d x d t$ over $\mathcal{U}_{L}$, for fixed initial data. There exists at least one optimal set and we provide some results on its regularity properties. In view of practical issues, it is far more interesting to consider then the problem of maximizing the observability constant. But this problem is difficult and we propose a slightly different approach which is actually more relevant for applications. We define the notion of a randomized observability constant, where this
\end{abstract}

*IRMAR, ENS Cachan Bretagne, Univ. Rennes 1, CNRS, UEB, av. Robert Schuman, 35170 Bruz, France (yannick.privat@bretagne.ens-cachan.fr).

†Université Pierre et Marie Curie (Univ. Paris 6) and Institut Universitaire de France, CNRS UMR 7598, Laboratoire Jacques-Louis Lions, F-75005, Paris, France (emmanuel.trelat@upmc.fr).

${ }^{\ddagger}$ BCAM - Basque Center for Applied Mathematics, Mazarredo, 14 E-48009 BilbaoBasque Country-Spain.

§Ikerbasque, Basque Foundation for Science, Alameda Urquijo 36-5, Plaza Bizkaia, 48011, Bilbao-Basque Country-Spain (zuazua@bcamath.org). 
constant is defined as an averaged over all possible randomized initial data. This constant appears as a spectral functional which is an eigenfunction concentration criterion. It can be also interpreted as a time asymptotic observability constant. This maximization problem happens to be intimately related with the ergodicity properties of the domain $\Omega$. We are able to compute the optimal value under strong ergodicity properties on $\Omega$ (namely, Quantum Unique Ergodicity). We then provide comments on ergodicity issues, on the existence of an optimal set, and on spectral approximations.

\section{The optimal observability problems}

Let $T>0$ fixed, and let $\Omega \subset \mathbb{R}^{n}$ be a bounded open connected set. We consider in parallel the wave equation

$$
\frac{\partial^{2} y}{\partial t^{2}}=\triangle y
$$

and the Schrödinger equation

$$
i \frac{\partial y}{\partial t}=\triangle y
$$

posed in $\Omega$, with Dirichlet boundary conditions. For any measurable subset $\omega \subset \Omega$, we observe the restrictions of the solutions of (1) and of (2) to $\omega$.

For the wave equation, the notion of observability is the following. For all $\left(y^{0}, y^{1}\right) \in L^{2}(\Omega, \mathbb{C}) \times H^{-1}(\Omega, \mathbb{C})$, there exists a unique solution $y \in$ $C^{0}\left(0, T ; L^{2}(\Omega, \mathbb{C})\right) \cap C^{1}\left(0, T ; H^{-1}(\Omega, \mathbb{C})\right)$ of $(1)$ such that $y(0, \cdot)=y^{0}(\cdot)$ and $y_{t}(0, \cdot)=y^{1}(\cdot)$. We say that an observability inequality holds whenever there exists $C>0$ such that

$$
C\left\|\left(y^{0}, y^{1}\right)\right\|_{L^{2}(\Omega, \mathbb{C}) \times H^{-1}(\Omega, \mathbb{C})}^{2} \leqslant \int_{0}^{T} \int_{\omega}|y(t, x)|^{2} d x d t,
$$

for all $\left(y^{0}, y^{1}\right) \in L^{2}(\Omega, \mathbb{C}) \times H^{-1}(\Omega, \mathbb{C})$. It is well known that within the class of $\mathcal{C}^{\infty}$ domains $\Omega$, this observability property holds if the pair $(\omega, T)$ satisfies the Geometric Control Condition in $\Omega$ (see [2]), according to which every ray of geometrical optics that propagates in $\Omega$ and is reflected on its boundary $\partial \Omega$ intersects $\omega$ within time $T$. We define the observability constant by

$$
C_{T}^{(W)}\left(\chi_{\omega}\right)=\inf \left\{\frac{\int_{0}^{T} \int_{\omega}|y(t, x)|^{2} d x d t}{\left\|\left(y^{0}, y^{1}\right)\right\|_{L^{2}(\Omega, \mathbb{C}) \times H^{-1}(\Omega, \mathbb{C})}^{2}} \mid\left(y^{0}, y^{1}\right) \in L^{2}(\Omega, \mathbb{C}) \times H^{-1}(\Omega, \mathbb{C}) \backslash\{(0,0)\}\right\} .
$$


For the Schrödinger equation, the observability goes as follows. For every $y^{0} \in L^{2}(\Omega, \mathbb{C})$, there exists a unique solution $y \in C^{0}\left(0, T ; L^{2}(\Omega, \mathbb{C})\right)$ of $(2)$ such that $y(0, \cdot)=y^{0}(\cdot)$. The system is said observable whenever there exists $C>0$ such that

$$
C\left\|y^{0}\right\|_{L^{2}(\Omega, \mathbb{C})}^{2} \leqslant \int_{0}^{T} \int_{\omega}|y(t, x)|^{2} d x d t
$$

for every $y^{0} \in L^{2}(\Omega, \mathbb{C})$. If there exists $T^{*}$ such that the pair $\left(\omega, T^{*}\right)$ satisfies the Geometric Control Condition then the observability inequality (5) holds for every $T>0$ (see [16]). We define the observability constant by

$$
C_{T}^{(S)}\left(\chi_{\omega}\right)=\inf \left\{\frac{\int_{0}^{T} \int_{\omega}|y(t, x)|^{2} d x d t}{\left\|y^{0}\right\|_{L^{2}(\Omega, \mathbb{C})}^{2}} \mid y^{0} \in L^{2}(\Omega, \mathbb{C}) \backslash\{0\}\right\} .
$$

In the sequel we fix $L \in(0,1)$, and we define the set

$\mathcal{U}_{L}=\left\{\chi_{\omega} \mid \omega\right.$ is a measurable subset of $\Omega$ of Lebesgue measure $\left.|\omega|=L|\Omega|\right\}$,

where $\chi_{\omega}$ is the characteristic function of $\omega$. Our objective is to maximize the observability properties of the wave or Schrödinger equation over the class $\mathcal{U}_{L}$, in a certain sense. We consider the two following mathematical problems.

\section{First problem (fixed initial data).}

- Wave equation $(1)$ : given $\left(y^{0}, y^{1}\right) \in L^{2}(\Omega, \mathbb{C}) \times H^{-1}(\Omega, \mathbb{C})$, we investigate the problem of maximizing the functional

$$
G_{T}\left(\chi_{\omega}\right)=\int_{0}^{T} \int_{\omega}|y(t, x)|^{2} d x d t
$$

over $\mathcal{U}_{L}$, where $y \in C^{0}\left(0, T ; L^{2}(\Omega, \mathbb{C})\right) \cap C^{1}\left(0, T ; H^{-1}(\Omega, \mathbb{C})\right)$ is the solution of $(1)$ such that $y(0, \cdot)=y^{0}(\cdot)$ and $\frac{\partial y}{\partial t}(0, \cdot)=$ $y^{1}(\cdot)$.

- Schrödinger equation (2): given $y^{0} \in L^{2}(\Omega, \mathbb{C})$, we investigate the problem of maximizing the functional $G_{T}$ defined by $(7)$ over $\mathcal{U}_{L}$, where $y \in C^{0}\left(0, T ; L^{2}(\Omega, \mathbb{C})\right)$ is the solution of (2) such that $y(0, \cdot)=y^{0}(\cdot)$. 
This first problem is the simplest possible and is interesting to be analyzed from the mathematical point of view, but in view of practical issues it is necessary to define an optimization problem that is independent on the initial data. Indeed, this kind of problem is motivated e.g. by the application to the optimal placement of sensors, in view of reconstructing some environment by means of local measures. The most natural criterion is then the observability constant itself, defined by (4) or by (6). But, firstly, the problem of maximizing (4) or $(4)$ over $\mathcal{U}_{L}$ happens to be very difficult ${ }^{1}$, and secondly, the observability constant, defined by an infimum, is probably a too much pessimistic criterion in practice: indeed when an engineer realizes a large number of measures (and would like these measures to be of high quality as far as possible), it is probable that only a few number of them will be "of low quality", corresponding to the observability constant.

In view of this remark, it makes sense to consider rather an averaged version of the observability inequality over randomized initial data. The procedure that we propose here goes as follows. It is inspired by the works of N. Burq and N. Tzvetkov on nonlinear partial differential equations with random initial data (see $[4,5])$.

Let $\left(\phi_{j}\right)_{j \in \mathbb{N}^{*}}$ be a Hilbertian basis of $L^{2}(\Omega)$ consisting of eigenfunctions of the Dirichlet Laplacian operator on $\Omega$, associated with the negative eigenvalues $\left(-\lambda_{j}^{2}\right)_{j \in \mathbb{N}^{*}}$. For all initial data $\left(y^{0}, y^{1}\right) \in L^{2}(\Omega, \mathbb{C}) \times H^{-1}(\Omega, \mathbb{C})$, the corresponding solution $y(1)$ can be expanded as

$$
y(t, x)=\sum_{j=1}^{+\infty}\left(a_{j} e^{i \lambda_{j} t}+b_{j} e^{-i \lambda_{j} t}\right) \phi_{j}(x),
$$

where

$$
\begin{aligned}
a_{j} & =\frac{1}{2}\left(\int_{\Omega} y^{0}(x) \phi_{j}(x) d x-\frac{i}{\lambda_{j}} \int_{\Omega} y^{1}(x) \phi_{j}(x) d x\right), \\
b_{j} & =\frac{1}{2}\left(\int_{\Omega} y^{0}(x) \phi_{j}(x) d x+\frac{i}{\lambda_{j}} \int_{\Omega} y^{1}(x) \phi_{j}(x) d x\right) .
\end{aligned}
$$

for every $j \in \mathbb{N}^{*}$. Note that $\left(a_{j}\right)_{j \in \mathbb{N}^{*}}$ and $\left(b_{j}\right)_{j \in \mathbb{N}^{*}}$ belong to $\ell^{2}(\mathbb{C})$ and that $\left\|\left(y^{0}, y^{1}\right)\right\|_{L^{2} \times H^{-1}}^{2}=2 \sum_{j=1}^{+\infty}\left(\left|a_{j}\right|^{2}+\left|b_{j}\right|^{2}\right)$. Following Burq and Tzvetkov, we now randomize some given initial data $\left(y^{0}, y^{1}\right)$ as follows. Let $\left(\beta_{1, j}^{\nu}\right)_{j \in \mathbb{N}^{*}}$ and

\footnotetext{
${ }^{1}$ It is similar to the well-known open problem of determining what are the best constants in Ingham's inequalities.
} 
$\left(\beta_{2, j}^{\nu}\right)_{j \in \mathbb{N}^{*}}$ two sequences of random laws on a probability space $(X, \mathcal{A}, \mathbb{P})$ that are independent, identically distributed with a common distribution $\theta$ for which there exists $c>0$ such that $\int_{\mathbb{R}} \mathrm{e}^{\gamma x} d \theta(x) \leqslant \mathrm{e}^{c \gamma^{2}}$. For example, Bernoulli or gaussian random laws can be considered. For every $\nu \in X$, we set

$y_{\nu}^{0}(t, \cdot)=\sum_{j=1}^{+\infty}\left(\beta_{1, j}^{\nu} a_{j}+\beta_{2, j}^{\nu} b_{j}\right) \phi_{j}(\cdot), \quad y_{\nu}^{1}(t, \cdot)=\sum_{j=1}^{+\infty} i \lambda_{j}\left(\beta_{1, j}^{\nu} a_{j}-\beta_{2, j}^{\nu} b_{j}\right) \phi_{j}(\cdot)$,

so that the corresponding solution of $(1)$ is

$$
y_{\nu}(t, \cdot)=\sum_{j=1}^{+\infty}\left(\beta_{1, j}^{\nu} a_{j} e^{i \lambda_{j} t}+\beta_{2, j}^{\nu} b_{j} e^{-i \lambda_{j} t}\right) \phi_{j}(\cdot)
$$

Then, instead of considering the deterministic observability inequality (3), we define the randomized observability inequality

$$
C_{T, \text { rand }}^{(W)}\left(\chi_{\omega}\right)\left\|\left(y^{0}, y^{1}\right)\right\|_{L^{2}(\Omega, \mathbb{C}) \times H^{-1}(\Omega, \mathbb{C})}^{2} \leqslant \mathbb{E}\left(\int_{0}^{T} \int_{\omega}\left|y_{\nu}(t, x)^{2}\right| d x d t\right),
$$

where

$$
C_{T, \text { rand }}^{(W)}\left(\chi_{\omega}\right)=\frac{1}{2} \inf _{\substack{\left(a_{j}\right),\left(b_{j}\right) \in \ell^{2}(\mathbb{C}) \\ \sum_{j=1}^{+\infty}\left(\left|a_{j}\right|^{2}+\left|b_{j}\right|^{2}\right)=1}} \mathbb{E}\left(\int_{0}^{T} \int_{\omega}\left|\sum_{j=1}^{+\infty}\left(\beta_{1, j}^{\nu} a_{j} e^{i \lambda_{j} t}+\beta_{2, j}^{\nu} b_{j} e^{-i \lambda_{j} t}\right) \phi_{j}(x)\right|^{2} d x d t\right),
$$

The same procedure is made for the Schrödinger equation, leading to define the randomized observability constant

$$
C_{T, \text { rand }}^{(S)}\left(\chi_{\omega}\right)=\inf _{\substack{\left(c_{j}\right) \in \ell^{2}(\mathbb{C}) \\ \sum_{j=1}^{+\infty}\left|c_{j}\right|^{2}=1}} \mathbb{E}\left(\int_{0}^{T} \int_{\omega}\left|\sum_{j=1}^{+\infty} \beta_{j}^{\nu} c_{j} e^{i \mu_{j} t} \phi_{j}(x)\right|^{2} d x d t\right)
$$

We have the following result.

Theorem 1. [[20]] For every measurable subset $\omega$ of $\Omega$, there holds

$$
2 C_{T, \text { rand }}^{(W)}\left(\chi_{\omega}\right)=C_{T, \text { rand }}^{(S)}\left(\chi_{\omega}\right)=T \inf _{j \in \mathbb{N}^{*}} \int_{\omega} \phi_{j}(x)^{2} d x .
$$


This motivates the consideration of the second problem below.

Second problem. We investigate the problem of maximizing the functional

$$
J\left(\chi_{\omega}\right)=\inf _{j \in \mathbb{N}^{*}} \int_{\omega} \phi_{j}(x)^{2} d x
$$

over $\mathcal{U}_{L}$

This criterion is a spectral energy (de)concentration one. Our main contribution is to provide evidence of the intimate relations between this problem and strong ergodicity properties of the set $\Omega$.

This second problem can be motivated in another way, by considering the following time averaging procedure. First of all, note that, for all initial data $\left(y^{0}, y^{1}\right) \in L^{2}(\Omega, \mathbb{C}) \times H^{-1}(\Omega, \mathbb{C})$, the quantity

$$
\frac{1}{T} \int_{0}^{T} \int_{\omega}|y(t, x)|^{2} d x d t
$$

where $y$ is the corresponding solution of the wave equation (1), has a limit as $T$ tends to $+\infty$. This leads to define the time asymptotic observability inequality

$$
C_{\infty}^{(W)}\left(\chi_{\omega}\right)\left\|\left(y^{0}, y^{1}\right)\right\|_{L^{2}(\Omega, \mathbb{C}) \times H^{-1}(\Omega, \mathbb{C})}^{2} \leqslant \lim _{T \rightarrow+\infty} \frac{1}{T} \int_{0}^{T} \int_{\omega}\left|y(t, x)^{2}\right| d x d t,
$$

where

$$
C_{\infty}^{(W)}\left(\chi_{\omega}\right)=\inf \left\{\lim _{T \rightarrow+\infty} \frac{1}{T} \frac{\int_{0}^{T} \int_{\omega}|y(t, x)|^{2} d x d t}{\left\|\left(y^{0}, y^{1}\right)\right\|_{L^{2} \times H^{-1}}^{2}} \mid\left(y^{0}, y^{1}\right) \in L^{2} \times H^{-1} \backslash\{(0,0)\}\right\} .
$$

Similarly, for the Schrödinger equation, we define

$$
C_{\infty}^{(S)}\left(\chi_{\omega}\right)=\inf \left\{\lim _{T \rightarrow+\infty} \frac{1}{T} \frac{\int_{0}^{T} \int_{\omega}|y(t, x)|^{2} d x d t}{\left\|y^{0}\right\|_{L^{2}(\Omega, \mathbb{C})}^{2}} \mid y^{0} \in L^{2}(\Omega, \mathbb{C}) \backslash\{0\}\right\} .
$$

We have the following result.

Theorem 2. [20] Assume that the domain $\Omega$ is such that every eigenvalue of the Dirichlet Laplacian operator is simple. For every measurable subset $\omega$ of $\Omega$, there holds

$$
2 C_{\infty}^{(W)}\left(\chi_{\omega}\right)=C_{\infty}^{(S)}\left(\chi_{\omega}\right)=\inf _{j \in \mathbb{N}^{*}} \int_{\omega} \phi_{j}(x)^{2} d x=J\left(\chi_{\omega}\right) .
$$


Note that the assumption of the simplicity of the spectrum of the Dirichlet Laplacian is generic with respect to the domain $\Omega$.

It can be observed that, although such optimal design problems have been widely investigated in the engineering literature (see e.g. [14, 17, 22] and references therein), few mathematical works exist that provide a theoretical rigorous analysis. We quote the remarkable articles $[11,12]$, where the problem of optimal domain is studied for the stabilization of one-dimensional wave equations with localized damping. These articles were actually the starting point of our own analysis. They are based on a thorough investigation through spectral considerations. In [18] we investigated the second problem presented previously in the one-dimensional case. We also quote the article [19] where we study the related problem of finding the optimal location of the support of the control for the one-dimensional wave equation. We refer to [20] for other references to related problems.

\section{First problem}

In this section, we consider fixed initial data $\left(y^{0}, y^{1}\right) \in L^{2}(\Omega) \times H^{-1}(\Omega)$ (resp., $y^{0} \in L^{2}(\Omega)$ ) for the wave equation (1) (resp., for the Schrödinger equation $(2)$ ), whose Fourier coefficients are defined by (9). Plugging (8) into (7) leads to

$$
G_{T}\left(\chi_{\omega}\right)=\int_{0}^{T} \int_{\omega}|y(t, x)|^{2} d x d t=\sum_{j, k=1}^{+\infty} \alpha_{j k} \int_{\omega} \phi_{i}(x) \phi_{j}(x) d x
$$

where

$$
\alpha_{j k}=\int_{0}^{T}\left(a_{j} e^{i \lambda_{j} t}+b_{j} e^{-i \lambda_{j} t}\right)\left(\bar{a}_{k} e^{-i \lambda_{k} t}+\bar{b}_{k} e^{i \lambda_{k} t}\right) d t .
$$

The coefficients $\alpha_{j k},(j, k) \in\left(\mathbb{N}^{*}\right)^{2}$, depend only on the initial data $\left(y^{0}, y^{1}\right)$, and can be easily computed as

$$
\begin{aligned}
\alpha_{j k}= & \frac{2 a_{j} \bar{a}_{k}}{\lambda_{j}-\lambda_{k}} \sin \left(\left(\lambda_{j}-\lambda_{k}\right) \frac{T}{2}\right) e^{i\left(\lambda_{j}-\lambda_{k}\right) \frac{T}{2}}+\frac{2 a_{j} \bar{b}_{k}}{\lambda_{j}+\lambda_{k}} \sin \left(\left(\lambda_{j}+\lambda_{k}\right) \frac{T}{2}\right) e^{i\left(\lambda_{j}+\lambda_{k}\right) \frac{T}{2}} \\
& +\frac{2 b_{j} \bar{a}_{k}}{\lambda_{j}+\lambda_{k}} \sin \left(\left(\lambda_{j}+\lambda_{k}\right) \frac{T}{2}\right) e^{-i\left(\lambda_{j}+\lambda_{k}\right) \frac{T}{2}}+\frac{2 b_{j} \bar{b}_{k}}{\lambda_{j}-\lambda_{k}} \sin \left(\left(\lambda_{j}-\lambda_{k}\right) \frac{T}{2}\right) e^{-i\left(\lambda_{j}-\lambda_{k}\right) \frac{T}{2}}
\end{aligned}
$$


whenever $\lambda_{j} \neq \lambda_{k}$, and

$$
\alpha_{j k}=T\left(a_{j} \bar{a}_{k}+b_{j} \bar{b}_{k}\right)+\frac{\sin \left(\lambda_{j} T\right)}{\lambda_{j}}\left(a_{j} \bar{b}_{k} e^{i \lambda_{j} T}+b_{j} \bar{a}_{k} e^{-i \lambda_{j} T}\right) .
$$

whenever $\lambda_{j}=\lambda_{k}$. We define the integrable function on $\Omega$

$$
\varphi(x)=\int_{0}^{T}|y(t, x)|^{2} d t=\sum_{i, j=1}^{+\infty} \alpha_{i j} \phi_{i}(x) \phi_{j}(x) .
$$

Then, from (17), there holds

$$
G_{T}\left(\chi_{\omega}\right)=\int_{\omega} \varphi(x) d x
$$

for every measurable subset $\omega$ of $\Omega$. With this expression, solving the first problem, that is the problem of maximizing the functional $G_{T}$ over $\mathcal{U}_{L}$, is quite immediate and we have the following result.

Theorem 3. [20] There exists at least one measurable subset $\omega$ of $\Omega$, solution of the first problem, characterized as follows. There exists a real number $\lambda$ such that every optimal set $\omega$ is contained in the level set $\{\varphi \geqslant \lambda\}$.

Moreover, if $\Omega$ has a $C^{\infty}$ boundary and if there exists $R>0$ such that

$$
\sum_{j=0}^{+\infty} \frac{R^{j}}{j !}\left(\left\|A^{j} y^{0}\right\|_{L^{2}}^{2}+\left\|A^{j-1} y^{1}\right\|_{L^{2}}^{2}\right)^{1 / 2}<+\infty,
$$

in the case of the wave equation, and

$$
\sum_{j=0}^{+\infty} \frac{R^{j}}{j !}\left\|A^{j} y^{0}\right\|_{L^{2}}<+\infty
$$

in the case of the Schrödinger equation, where $A=\sqrt{-\triangle}$ (square root of the Dirichlet-Laplacian), then the first problem has a unique ${ }^{2}$ solution $\chi_{\omega} \in \mathcal{U}_{L}$, satisfying moreover the following properties:

\footnotetext{
${ }^{2}$ Similarly to the definition of elements of $L^{\infty}$, the subset $\omega$ is unique within the class of all measurable subsets of $\Omega$ quotiented by the set of all measurable subsets of $\Omega$ of zero Lebesgue measure.
} 
- there exists $\eta>0$ such that $d(\omega, \partial \Omega)>\eta$, where $d$ denotes the Euclidean distance;

- $\omega$ has a finite number of connected components;

- if $\Omega$ is symmetric with respect to an hyperplane, then $\omega$ enjoys the same symmetry property.

Remark 1. The conditions (23) or (24) stipulate that the initial data share analyticity properties (note that they imply that $y^{0}$ and $y^{1}$ are analytic on $\Omega$.

Remark 2. The optimal solution may not be unique whenever the function $\varphi$ is constant on some subset of $\Omega$ of positive measure. If $\varphi$ is constant equal to $c$ on some subset $I \subset \Omega$ of positive measure, and if $|\{\varphi \geqslant c\}|<L|\Omega|<$ $|\{\varphi>c\}|$, then there exists an infinite number of measurable subsets $\omega \subset \Omega$ maximizing (22), all of them containing the subset $\{\varphi>c\}$. The part of $\omega$ lying in $\{\varphi=c\}$ can indeed be chosen arbitrarily.

Remark 3. In the one-dimensional case $\Omega=[0, \pi]$, one has $\phi_{j}(x)=\sqrt{\frac{2}{\pi}} \sin (j x)$ and $\lambda_{j}=j$ for every $j \in \mathbb{N}^{*}$. If moreover $T=2 p \pi$ with $p \in \mathbb{N}^{*}$, then $\alpha_{i j}=0$ whenever $i \neq j$, and $\alpha_{j j}=p \pi\left(\left|a_{j}\right|^{2}+\left|b_{j}\right|^{2}\right)$ for all $(i, j) \in\left(\mathbb{N}^{*}\right)^{2}$, and therefore

$$
G_{2 p \pi}\left(\chi_{\omega}\right)=\sum_{j=1}^{+\infty} \alpha_{j j} \int_{\omega} \sin ^{2}(j x) d x .
$$

Hence in that case the functional $G_{2 p \pi}$ does not involve any crossed terms. The second problem for this one-dimensional case was studied in details in [18]. In particular, in this case the non-uniqueness phenomenon can be exactly characterized in terms of Fourier series.

Remark 4. If we relax, even slightly, the analyticity assumptions (23), then the optimal optimal set $\omega$ is not necessarily unique and moreover may have an infinite number of connected components. In [20] we prove that, in the framework of Remark 3, there exist smooth initial data $\left(y^{0}, y^{1}\right)$ for the wave equation (resp., $y^{0}$ for the Schrödinger equation) defined on $[0, \pi]$ for which the optimal set $\omega$ has a fractal structure (it is of Cantor type) and thus in particular has an infinite number of connected components. 


\section{Second problem and quantum ergodicity}

The second problem

$$
\sup _{\chi_{\omega} \in \mathcal{U}_{L}} J\left(\chi_{\omega}\right)
$$

is posed on a set $\mathcal{U}_{L}$ that does not have nice compactness properties. It is usual in optimal design problems to consider a convexified version of the problem. Here, the convex closure of $\mathcal{U}_{L}$ for the weak star topology of $L^{\infty}$ is

$$
\overline{\mathcal{U}}_{L}=\left\{a \in L^{\infty}(\Omega,[0,1])\left|\int_{\Omega} a(x) d x=L\right| \Omega \mid\right\} .
$$

Replacing $\chi_{\omega} \in \mathcal{U}_{L}$ with $a \in \overline{\mathcal{U}}_{L}$, the convexified version of the second problem (26) is

$$
\sup _{a \in \overline{\mathcal{U}}_{L}} J(a)
$$

where

$$
J(a)=\inf _{j \in \mathbb{N}^{*}} \int_{\Omega} a(x) \phi_{j}(x)^{2} d x .
$$

Remark 5. Note that

$$
\sup _{\chi_{\omega} \in \mathcal{U}_{L}} \inf _{j \in \mathbb{N}^{*}} \int_{\Omega} \chi_{\omega}(x) \phi_{j}(x)^{2} d x \leqslant \sup _{a \in \overline{\mathcal{U}}_{L}} \inf _{j \in \mathbb{N}^{*}} \int_{\Omega} a(x) \phi_{j}(x)^{2} d x,
$$

but it is not obvious to see whether the inequality is strict or not. Indeed, since the functional $J$ under consideration fails to be lower semi-continuous, the usual $\Gamma$-convergence theory does not apply to our problem. This difficulty can be made evident in dimension one (see Remark 3). In that case, one has $\phi_{j}(x)=\sqrt{\frac{2}{\pi}} \sin (j x)$ for every $j \in \mathbb{N}^{*}$, and it is easy to see that $\sup _{a \in \overline{\mathcal{U}}_{L}} J(a)=L$ and that the supremum is reached with the constant function $a(\cdot)=L$. Besides, the sequence of functions $\chi_{\omega_{N}}$, where $\omega_{N}=\cup_{k=1}^{N}\left[\frac{k \pi}{N+1}-\frac{L \pi}{2 N}, \frac{k \pi}{N+1}+\frac{L \pi}{2 N}\right]$ for every $N \in \mathbb{N}^{*}$, converges to the constant function $a(\cdot)=L$ for the weak star topology of $L^{\infty}$, but nevertheless, computations show that $\lim \sup _{N \rightarrow+\infty} J\left(\chi_{\omega_{N}}\right)<L$.

In what follows, we make the following assumption on $\Omega$.

Weak Quantum Ergodicity (WQE) property. There exists a subsequence of $\left(\phi_{j}^{2}\right)_{j \in \mathbb{N}^{*}}$ converging to $\frac{1}{|\Omega|}$ in weak star $L^{\infty}$ topology. 
Under this assumption, there holds

$$
\sup _{a \in \overline{\mathcal{U}}_{L}} \inf _{j \in \mathbb{N}^{*}} \int_{\Omega} a(x) \phi_{j}(x)^{2} d x=L,
$$

and moreover the supremum is reached with the constant function $a=L$ on $\Omega$. Hence, from now on we have

$$
\sup _{\chi_{\omega} \in \mathcal{U}_{L}} \inf _{j \in \mathbb{N}^{*}} \int_{\Omega} \chi_{\omega}(x) \phi_{j}(x)^{2} d x \leqslant L,
$$

and we wonder whether this inequality is strict or not, or in other words, whether there is a gap or not between the second problem and its convexified version.

Remark 6. The WQE assumption is always true in dimension one, as obvious consequence of Lebesgue Lemma. The situation is more complicated in dimension greater than one and we have the following facts.

- In a hypercube, or in the unit ball of $\mathbb{R}^{n}$, WQE holds true.

- More generally, WQE follows from ergodicity properties. In [8, 10, 24] it is proved that, if the domain $\Omega$ is an ergodic billiard with piecewise smooth boundary, then the following property QE is satisfied.

Quantum Ergodicity (QE) property. There exists a subsequence of $\left(\phi_{j}^{2}\right)_{j \in \mathbb{N}^{*}}$ of density one converging to $\frac{1}{|\Omega|}$ in weak star $L^{\infty}$ topology.

Obviously, QE implies WQE. Actually the result (known as Shnirelman Theorem) is stronger: a density-one subsequence of the probability measures $\mu_{j}=\phi_{j}^{2} d x$ converges weakly to the Liouville measure of $\Omega$. Here the domain $\Omega$ is seen as a billiard where the flow moves at unit speed and bounces at the boundary according to the Geometric Optics laws.

To obtain a no-gap result, we need to strengthen WQE into the following assumption.

Quantum Unique Ergodicity (QUE) property. The whole sequence $\left(\phi_{j}^{2}\right)_{j \in \mathbb{N}^{*}}$ converges to $\frac{1}{|\Omega|}$ in weak star $L^{\infty}$ topology. 
Theorem 4. ([20]) If $\Omega$ satisfies $Q U E$, then

$$
\sup _{\chi_{\omega} \in \mathcal{U}_{L}} \inf _{j \in \mathbb{N}^{*}} \int_{\omega} \phi_{j}(x)^{2} d x=L
$$

for every $L \in(0,1)$. In other words, under QUE there is no gap between the problem (26) and its convexified version (28).

Remark 7. The QUE property is very well-known in quantum physics and mathematical physics. The quantity $\mu_{j}(\omega)=\int_{\omega} \phi_{j}^{2}(x) d x$ represents the probability of finding the quantum state in $\omega$.

Obviously, QUE implies QE. Note that the QE property lets open the possibility of having an exceptional subsequence of $\phi_{j}^{2}$ converging weakly to some other measure. It may indeed happen that a subsequence (necessarily of density zero under QE) converges to an invariant measure like for instance a measure carried by closed geodesics or invariant tori generated by such geodesics. This energy concentration phenomenon is referred to as a (strong) scar and up to now remains a widely unknown issue (see $[3,7])$.

The QUE assumption postulates that there is no such concentration phenomenon (see [23]), but very few results are known. In the one-dimensional case, QUE is always true. But in the multi-dimensional case, up to now no example is known where QUE holds (in the context of the Dirichlet-Laplacian). The ergodicity of $\Omega$ is however a necessary assumption for QUE to hold. Note that strictly convex billiards whose boundary is $C^{6}$ are not ergodic (see [15]), and there are sequences of positive density of eigenfunctions which concentrate on caustics. Rational polygonal billiards are not ergodic, but generic polygonal billiards are ergodic (see [13]). Recently, Hassell has shown in [9] that there exist some convex (stadium-shaped) sets satisfying QE but not QUE. Note that it is a longstanding conjecture that every compact negatively curved manifold should satisfy QUE (see [21]). Using a concept of entropy, it is proved in [1] that on a compact manifold with negative curvature the eigenfunctions cannot concentrate entirely on closed geodesics and at least half of their energy remains chaotic.

It is an open question of knowing whether the statement of Theorem 4 still holds true for domains satisfying QE or only WQE. Note however that we have the following corollary.

Corollary 1. Assume that $\Omega$ satisfies $Q E$. There exists a subset $\mathcal{I}$ of $\mathbb{N}^{*}$ of 
density 1 such that

$$
\sup _{\chi_{\omega} \in \mathcal{U}_{L}} \inf _{j \in \mathcal{I}} \int_{\omega} \phi_{j}(x)^{2} d x=L
$$

Remark 8. QUE is a sufficient condition implying the no-gap statement (33), but is not sharp: it is not a necessary condition. For instance the twodimensional square does not satisfy QUE (nor QE), but satisfies WQE, and in this configuration it can be easily shown that the no-gap result (33) holds.

Remark 9. The question of knowing whether the supremum in (33) is reached or not is unexpectedly difficult. We have a clear answer only in dimension one. We gather hereafter the results we have been able to prove.

- In the one-dimensional case $\Omega=[0, \pi]$, for $L \in(0,1)$, the supremum of $J$ over $\mathcal{U}_{L}$ (which is equal to $L$ ) is reached if and only if $L=1 / 2$. In that case, it is reached for all measurable subsets $\omega \subset[0, \pi]$ of measure $\pi / 2$ such that $\omega$ and its symmetric $\omega^{\prime}=\pi-\omega$ are disjoint and complementary in $[0, \pi]$.

- For the two-dimensional square $\Omega=[0, \pi]^{2}$, the supremum of $J$ over the class of all possible subsets $\omega=\omega_{1} \times \omega_{2}$ of Lebesgue measure $L \pi^{2}$, where $\omega_{1}$ and $\omega_{2}$ are measurable subsets of $[0, \pi]$, is reached if and only if $L \in\{1 / 4,1 / 2,3 / 4\}$. In that case, it is reached for all such sets $\omega$ satisfying

$$
\frac{1}{4}\left(\chi_{\omega}(x, y)+\chi_{\omega}(\pi-x, y)+\chi_{\omega}(x, \pi-y)+\chi_{\omega}(\pi-x, \pi-y)\right)=L,
$$

for almost all $(x, y) \in\left[0, \pi^{2}\right]$.

It is by the way interesting to note that there exist optimal sets not satisfying the Geometric Control Condition, and thus in this case the deterministic observability constant is equal to 0 , whereas $2 C_{T \text {,rand }}^{(W)}\left(\chi_{\omega}\right)=$ $C_{T, \text { rand }}^{(S)}\left(\chi_{\omega}\right)=T L$.

We conjecture that, if $\Omega$ is the unit $N$-dimensional hypercube, then there exists a finite number of values of $L \in(0,1)$ such that the the supremum in (33) is reached; for generic domains $\Omega$, there exists a finite number of values of $L \in(0,1)$ such that the the supremum in (33) is reached. 
Remark 10. As a final remark, let us stress that our main contribution is to put in evidence the intimate relations existing between shape optimization problems and the (strong) ergodicity properties of the domain $\Omega$ under consideration. It can be noticed that such a connection was suggested in the early work [6], where the authors were concerned with the question of determining sufficient conditions on dissipative terms added into a conservative equation, ensuring an exponential decay of the solutions. The role of the quantum effects of bouncing balls and of the whispering galleries was underlined in their study. Our results here provide clear statements making such a connection and suggest new issues in the analysis of optimal design problems.

We end the study of the second problem by considering the spectral truncation of the functional $J$ defined by (13)

$$
J_{N}\left(\chi_{\omega}\right)=\min _{1 \leqslant j \leqslant N} \int_{\omega} \phi_{j}(x)^{2} d x
$$

for every $N \in \mathbb{N}^{*}$ and every measurable subset $\omega$ of $\Omega$. The spectral approximation of the second problem is defined as

$$
\sup _{\chi_{\omega} \in \mathcal{U}_{L}} J_{N}\left(\chi_{\omega}\right)
$$

As before, the functional $J_{N}$ is extended to $\overline{\mathcal{U}}_{L}$ by

$$
J_{N}(a)=\min _{1 \leqslant j \leqslant N} \int_{\Omega} a(x) \phi_{j}(x)^{2} d x
$$

for every $a \in \overline{\mathcal{U}}_{L}$. We have the following result.

Theorem 5. 1. For every measurable subset $\omega$ of $\Omega$, the sequence $\left(J_{N}\left(\chi_{\omega}\right)\right)_{N \in \mathbb{N}^{*}}$ is nonincreasing and converges to $J\left(\chi_{\omega}\right)$.

2. There holds

$$
\lim _{N \rightarrow+\infty} \max _{a \in \overline{\mathcal{U}}_{L}} J_{N}(a)=\max _{a \in \overline{\mathcal{U}}_{L}} J(a) .
$$

Moreover, if $\left(a^{N}\right)_{n \in \mathbb{N}^{*}}$ is a sequence of maximizers of $J_{N}$ in $\overline{\mathcal{U}}_{L}$, then up to a subsequence $\left(a^{N}\right)_{n \in \mathbb{N}^{*}}$ converges to a maximizer of $J$ in $\overline{\mathcal{U}}_{L}$ in $L^{\infty}$ weak star topology. 
3. For every $N \in \mathbb{N}^{*}$, the problem (35) has a unique solution $\chi_{\omega^{N}}$, where $\omega^{N} \in \mathcal{U}_{L}$. Moreover, $\omega^{N}$ has a finite number of connected components.

The theorem allows one to construct a maximizing sequence for the second problem. Several numerical simulations are provided in [20].

Remark 11. Note however that the following property is proved in [18]. In the one-dimensional case $\Omega=[0, \pi], \omega^{N}$ is the union of at most $N$ intervals, is symmetric with respect to $\pi / 2$, and moreover there exists $L_{N} \in(0,1] \mathrm{such}$ that, for every $L \in\left(0, L_{N}\right]$, the optimal domain $\omega^{N}$ satisfies

$$
\int_{\omega^{N}} \sin ^{2} x d x=\int_{\omega^{N}} \sin ^{2}(2 x) d x=\cdots=\int_{\omega^{N}} \sin ^{2}(N x) d x .
$$

This technical property (quite difficult to prove), stated in [12, Theorem 3.2 ] but whose proof is not completely correct, causes the so-called spillover phenomenon, according to which the optimal domain $\omega^{N}$ solution of (35) with the $N$ first modes is the worst possible domain for the problem with the $N+1$ first modes. Actually, $\omega^{N}$ concentrates around the nodes $\frac{k \pi}{N+1}, k=1, \ldots, N$. This is rather a bad news for practical purposes, but is in accordance with the fact that the problem has no solution whenever $L \neq 1 / 2$.

\section{References}

[1] N. Anantharaman, Entropy and the localization of eigenfunctions, Ann. of Math. 2 (2008), Vol. 168, 435-475.

[2] C. Bardos, G. Lebeau, J. Rauch, Sharp sufficient conditions for the observation, control, and stabilization of waves from the boundary, SIAM J. Control Optim. 30 (1992), no. 5, 1024-1065.

[3] F. Bonechi, S. De Bièvre, Controlling strong scarring for quantized ergodic toral automorphisms, Duke Math. J. 117 (2003), no. 3, 571-587.

[4] N. Burq, Large-time dynamics for the one-dimensional Schrödinger equation, Proc. Roy. Soc. Edinburgh Sect. A. 141 (2011), no. 2, 227-251.

[5] N. Burq, N. Tzvetkov, Random data Cauchy theory for supercritical wave equations. I. Local theory, Invent. Math. 173 (2008), no. 3, 449475 . 
[6] G. Chen, S.A. Fulling, F.J. Narcowich, S. Sun, Exponential decay of energy of evolution equations with locally distributed damping, SIAM J. Appl. Math. 51 (1991), no. 1, 266-301.

[7] F. Faure, S. Nonnenmacher, S. De Bièvre, Scarred eigenstates for quantum cat maps of minimal periods, Comm. Math. Phys. 239 (2003), no. 3, 449-492.

[8] P. Gérard, E. Leichtnam, Ergodic properties of eigenfunctions for the Dirichlet problem, Duke Math. J. 71 (1993), 559-607.

[9] A. Hassell, Ergodic billiards that are not quantum unique ergodic, Ann. Math. (2) 171 (2010), 605-619.

[10] A. Hassell, S. Zelditch, Quantum ergodicity of boundary values of eigenfunctions, Comm. Math. Phys. 248 (2004), no. 1, 119-168.

[11] P. Hébrard, A. Henrot, Optimal shape and position of the actuators for the stabilization of a string, Syst. Cont. Letters 48 (2003), 199-209.

[12] P. Hébrard, A. Henrot, A spillover phenomenon in the optimal location of actuators, SIAM J. Control Optim. 44 2005, 349-366.

[13] S. Kerckhoff, H. Masur, J. Smillie, Ergodicity of billiard flows and quadratic differentials, Ann. of Math. (2) 124 (1986), no. 2, 293-311.

[14] S. Kumar, J.H. Seinfeld, Optimal location of measurements for distributed parameter estimation, IEEE Trans. Autom. Contr. 23(1978), 690-698.

[15] V.F. Lazutkin, On the asymptotics of the eigenfunctions of the Laplacian, Soviet Math. Dokl. 12 (1971), 1569-1572.

[16] G. Lebeau, Contrôle de l'equation de Schrödinger, J. Math. Pures Appl. 71 (1992), 267-291.

[17] K. Morris, Linear-quadratic optimal actuator location, IEEE Trans. Automat. Control 56 (2011), no. 1, 113-124.

[18] Y. Privat, E. Trélat, E. Zuazua, Optimal observability of the onedimensional wave equation, Preprint Hal (2012). 
[19] Y. Privat, E. Trélat, E. Zuazua, Optimal location of controllers for the one-dimensional wave equation, Preprint Hal (2012).

[20] Y. Privat, E. Trélat, E. Zuazua, Optimal observability of wave and Schrödinger equations in ergodic domains, ongoing work (2012).

[21] Z. Rudnick, P. Sarnak, The behaviour of eigenstates of arithmetic hyperbolic manifolds, Comm. Math. Phys. 161 (1994), no. 1, 195-213.

[22] M. van de Wal, B. Jager, A review of methods for input/output selection, Automatica 37 (2001), no. 4, 487-510.

[23] S. Zelditch, Note on quantum unique ergodicity, Proc. Amer. Math. Soc. 132 (2004), 1869-1872.

[24] S. Zelditch, M. Zworski, Ergodicity of eigenfunctions for ergodic billiards, Comm. Math. Phys. 175 (1996), no. 3, 673-682. 